\title{
Une grande satisfaction pour EXIT Suisse romande
}

Jérôme Sobel

Président d'Exit ADMD Suisse romande

Correspondance:

Docteur Jérôme Sobel Bellefontaine 2

CH-1003 Lausanne
Le 18 janvier 2013 Monsieur Pierre-Yves Maillard, Chef du Département de la santé et de l'action sociale, a publié dans la Feuille des avis officiels du canton de Vaud les directives d'application de l'article $27 \mathrm{~d}$ de la loi sur la santé publique sur l'assistance au suicide en établissement sanitaire reconnu d'intérêt public.

\section{Genèse du processus}

Suite à l'opposition de principe de certains EMS (Etablissement médico-social) à toutes assistances au suicide dans leurs murs et suite à des situations de blocage, l'association Exit ADMD Suisse romande (Association pour le droit à mourir dans la dignité) a déposé une initiative populaire législative intitulée «Assistance au suicide en EMS». Cette initiative déposée le 3 février 2009 voulait inscrire dans la loi cantonale l'obligation pour tous les EMS subventionnés de tolérer l'assistance au suicide requise par l'un pathologie psychiatrique dans ses antécédents ne doit pas être suspecté automatiquement de perte de discernement du seul fait de demander une assistance au suicide.

Dans sa chambre d'EMS, le résident dispose d'un lieu privé où il doit pouvoir faire valoir librement son droit et son choix.

Notre initiative souhaitait permettre l'autodétermination sans entrave des résidents en EMS qui pourraient en pleine conscience demander une assistance au suicide. Un résident peut être fragilisé sur le plan physique mais il ne doit pas être automatiquement taxé de manque de discernement. Ses droits personnels ne peuvent pas être réduits simplement parce qu'il vit en EMS et que l'équipe soignante estime irrecevable une demande qui ne correspond pas aux valeurs qu'elle défend. L'évaluation de la demande par l'équipe soignante sera problématique quant à sa neutralité et son objectivité.

\section{Le dialogue avec les EMS est devenu plus facile, dans le respect de chacun.}

de leurs résidents à l'intérieur de l'établissement. Elle ne fixait toutefois aucun cadre aux mesures d'accompagnement.

Le Conseil d'Etat et le Grand Conseil vaudois ont souhaité opposer à cette initiative un contre-projet pour préciser à quelles conditions une telle assistance au suicide serait possible, afin de prévenir d'éventuels abus.

\section{Argumentaire}

L'avis des initiants est basé sur un arrêt du Tribunal Fédéral du 3 novembre 2006 confirmant que chaque être humain capable de discernement a le droit garanti par la Constitution et la Convention européenne des droits de l'homme (CEDH) de décider de la manière et du moment de sa propre mort. Ce droit appartient au droit à l'autodétermination au sens de l'article 8 chiffre 1 de la CEDH et ceci aussi longtemps que la personne concernée est en situation de faire librement son choix et d'agir conformément à sa volonté.

Cet arrêt du TF est une clarification bienvenue et implique par conséquent qu'un malade atteint de pathologie organique et qui n'a jamais présenté de
Le contre-projet soutenu par le Conseil d'Etat permet de fixer clairement les conditions dans lesquelles les établissements sanitaires (hôpitaux et EMS) reconnus d'intérêt public ne peuvent refuser la tenue d'une assistance au suicide dans leurs murs. Ce contre-projet respecte la liberté individuelle et le droit à l'autodétermination. Il protège d'une prise de décision précipitée en instaurant un accompagnement et une procédure garantissant que la demande d'assistance correspond bien à la volonté libre (sans pression d'autrui) et réfléchie du résident ou patient. Il assure également que toutes les alternatives, en particulier celle des soins palliatifs, ont été envisagées, afin de garantir que le suicide assisté constitue véritablement la solution de dernier recours souhaitée par la personne.

$\mathrm{Au}$ contraire de l'initiative, le contre-projet du Conseil d'Etat tient compte du fait qu'un EMS n'est pas seulement un équivalent du domicile d'un ou d'une résident-e, mais qu'il est également un lieu de soins et de vie communautaire, accueillant des personnes fragilisées, entourées par du personnel soignant. Il permet dès lors, tout en garantissant le droit à l'autodétermination de la personne, de clarifier le 


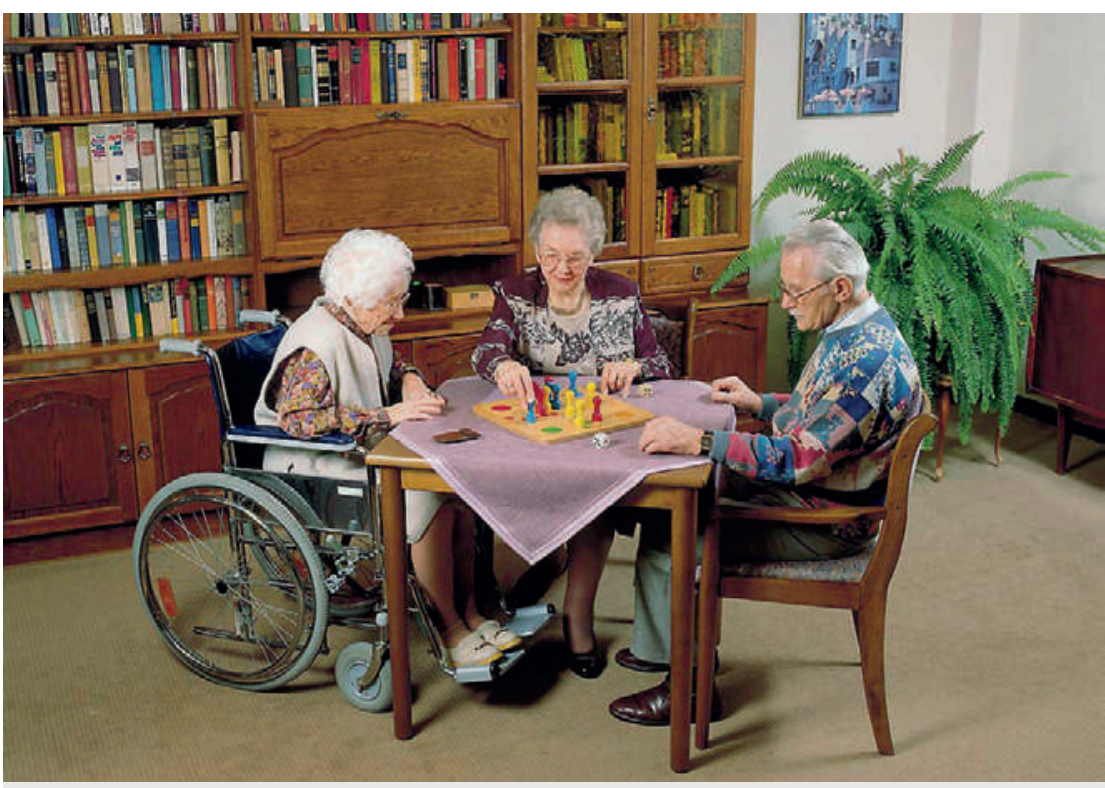

Les droits personnels d'un résident ne peuvent pas être réduits simplement parce qu'il vit en EMS.

rôle de l'EMS et de son personnel face à des demandes d'assistance au suicide. Le Conseil d'Etat estime qu'il est inapproprié de réduire le souci du respect de l'intégrité des résidents, des patients et des soignants à des complications administratives. Il considère devoir garantir la protection des personnes malades ou âgées qui sont vulnérables.

Contrairement aux initiants, le Conseil d'Etat propose d'introduire un cadre clair également dans les hôpitaux reconnus d'intérêt public.

\section{Votations}

Exit Suisse romande a maintenu son projet car l'association considérait a priori le contre-projet comme une mise sous tutelle des patients. Exit craignait qu'on puisse utiliser ce contre-projet pour bloquer les demandes d'assistance au suicide et les égarer dans un labyrinthe administratif.

Le 17 juin 2012 la population vaudoise a voté en faveur du contre-projet par $60 \%$ contre $39 \%$ au projet d'Exit. Les personnes qui prônaient un refus aux deux objets ont été balayées. La population vaudoise a ainsi clairement montré qu'elle soutient le principe de l'assistance au suicide, mais dans un cadre contrôlé et avec une concertation entre les intervenants.

Fort de ce résultat, le Département de la santé et de l'action sociale a pu encore préciser et compléter par des directives la base légale qu'est l'un article $27 \mathrm{~d}$ de la loi sur la santé publique.

\section{Ajouts et précisions des directives entrées en vigueur le $1^{\mathrm{er}}$ janvier 2013}

Un délai maximum de 4 semaines a été imparti au médecin responsable de l'établissement sanitaire pour qu'il se détermine quant à l'acceptation d'une demande d'assistance au suicide d'un patient à l'hôpital ou d'un résident en EMS, selon la complexité du cas. S'il suspecte une perte de discernement, il lui incombe d'en apporter la preuve en sollicitant l'avis d'un psychiatre. En cas de désaccord quant aux conclusions, le patient/résident a maintenant la possibilité de saisir le bureau de la médiation et le médecin cantonal ou la commission des plaintes des patients.

Ces possibilités de recours du patient/résident n'avaient pas été évoquées dans le cadre de la votation et le fait qu'elles soient maintenant inscrites dans la loi a contribué à modifier la perception de l'association Exit Suisse romande quant au contreprojet accepté par le peuple.

\section{Application de la procedure legale}

Depuis la votation du 17 juin 2012 et l'adoption du contre-projet, l'association Exit Suisse romande a suivi les procédures décrites et elle ne peut que s'en féliciter. Le dialogue avec les EMS est devenu plus facile, dans le respect de chacun. Nous avons pu procéder à 16 assistances au suicide en EMS en Suisse romande. Nous sommes même intervenus dans un cas, intra muros, au CHUV à Lausanne.

Les débats publics qui ont précédé la votation du 17 juin ont permis une réflexion et une ouverture d'esprit qui va bien au-delà du seul canton de Vaud. Le nombre de nouveaux adhérents à l'association Exit ADMD Suisse romande a par ailleurs fortement augmenté.

\section{Quelques chiffres}

Exit Suisse romande compte actuellement près de 18000 membres. En 2012 notre association a reçu 258 demandes d'assistances au suicide et nous avons aidé 144 personnes à mourir. 12 demandes ont été refusées; nous sommes une barrière contre des suicides émotionnels pour de mauvaises raisons chez les jeunes.

102 personnes que nous avons rencontrées ont été sécurisées par notre visite car elles ont été entendues et comprises. Ces personnes savent que si elles nous sollicitent ultérieurement pour fixer une date à leur auto-délivrance, nous les aiderons.

Nos membres sont ainsi rassurés et certains mourront de leur mort naturelle sans avoir besoin de notre aide. Exit permet ainsi à ces personnes de mieux vivre leur fin de vie car nous sommes par notre seule présence aussi efficaces que des psychothérapies de soutien ou des médicaments anti-dépresseurs; nos adhérents savent que notre association est crédible et fiable. Nous tenons nos promesses, l'association fait ce qu'elle dit et dit ce qu'elle fait.

Le tabou du suicide médicalement assisté doit être brisé dans nos sociétés cantonales de médecine ainsi que dans nos facultés. L'enseignement de cette problématique devrait faire partie du cursus de formation médicale. Nos jeunes confrères pourraient ainsi acquérir, s'ils le souhaitent, un savoir faire et un savoir être et ce pour le plus grand bien de chacun. La mort est un événement qui mérite d'être préparé et qui peut être approché dans un climat de sérénité. 